\title{
Instability of a liquid jet in high velocity coaxial air stream
}

\author{
Jun-jie Wang ${ }^{1 a}$, Li-jun Yang ${ }^{2 b}$,Qing-fei Fu ${ }^{3 c}$ and Run-ze Duan \\ 1,2,3 Beijing University of Aeronautics and Astronautics, Beijing, China, 100191 \\ aemail:junjiebuaa@126.com
}

Keywords: liquid jet, linear instability, explicit solution

Abstract. This paper reports an approach to derive the explicit solutions of the dispersion equation of a liquid jet with small viscosity in high velocity coaxial air stream. The dispersion equation, which contains the relationship of the growth rate and wavenumber, is very complex when taking the viscosity into account. Researchers have to use the numerical method to analyze the disturbance on the jet surface. The explicit solution is more concise to understand the effect of physical parameters on the jet breakup than the numerical one, and the results show that the explicit solution is applicable for small viscosity liquid jet in high velocity air streams.

\section{Introduction}

The atomization of liquid jets with high velocity gas streams is an important process in many technical applications. The liquid jet emanates from the central orifice with relatively lower speed compared to the co-flowing gas. In this process, the surface of the liquid phase becomes unstable, and then waves formed on the liquid surface, are amplified, and shed ligaments that rapidly collapse to form drops.

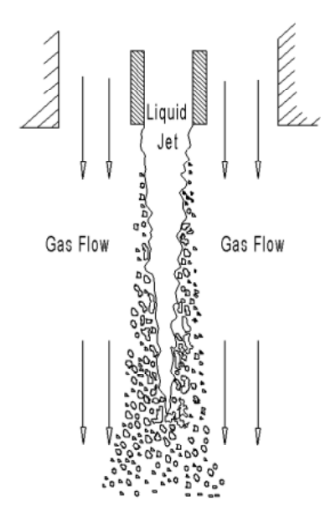

Fig. 1 Schematic of the air-blast atomization

Owing to the complexity of the physical phenomena involved in the process, the physical mechanisms are still not well understood. The study of liquid atomization in the high velocity gas flow is pursued usually by experiments.

As well, some researchers tried to present theoretical approaches for the problem of the liquid jet breakup in the high velocity regime. Aerodynamic effect is the most developed theory of the atomization. The growth of disturbances, generating either within the atomizer or due to aerodynamic interaction with the surrounding gas, is mainly responsible for the formation of ligaments which ultimately breaks up to produce smaller, more stable liquid globes.

A linear stability analysis is presented for a liquid jet that includes the effects of the 
surrounding gas, surface tension and the liquid viscosity on the wave growth process. Rayleigh ${ }^{[1]}$ conducted the classical linear stability analysis of the inviscid liquid jet, and found that axisymmetric disturbances are the dominant instability mode that leads to the breakup. Levich ${ }^{[2]}$ considered the case of viscosity, and proposed that atomization is due to aerodynamic interaction between the liquid and the gas leading to unstable wave growth on the liquid jet surface. Reitz and Bracco $^{[3]}$ presented a unified first-order linear steady theory to analyze the breakup of the liquid jet, and to predict the spray angle. They assumed that the most unstable wave conducts the breakup process, and then conducted a detailed experimental investigation where spray angle was measured for different operating conditions. $\operatorname{Lin}^{[4]}$ developed the linear stability analysis of a viscous liquid jet with respect to spatially growing disturbances. $\mathrm{Li}^{[5]}$ derived that a viscous liquid jet in an inviscid gas medium with three-dimensional disturbances by linear stability analysis. And it was found that the most unstable wave which has the most unstable growth rate conducted the breakup process.

Owing to the complexity of the dispersion equation, the numerical solution have been taken to analyze the principle of the liquid jet breakup. However, the numerical solution fails to tell the role of the different physical parameters in the process. In contrast, the present explicit solution is expressed as functions of gas velocity, liquid velocity and other physical characteristics. This paper just focuses on this problem and proposed an approach to derive the explicit solution on the small viscosity liquid jet in high velocity air stream.

\section{Linear stability analysis}

A viscous incompressible liquid jet with a radius $r_{0}$, density $\rho_{l}$, surface tension $\sigma$, viscosity $\mu$ and uniform velocity $u_{l}$, moving through an inviscid gas of destiny $\rho_{g}$ and uniform velocity $u_{g}$, is considered, as depicted in Fig.2. Note that $u_{g} u_{l}$, a relative velocity $u^{g}$, defined as $u^{g}=u_{g}-u_{l}$ is applied. The polar coordinate system, moving with the jet, is fixed at the centerline of the jet at the orifice.

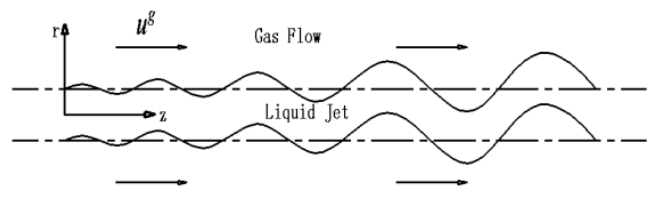

Fig. 2 Schematic of a spectrum of disturbances on the jet surface

The present stability analysis for a liquid jet, which includes the effects of the surrounding gas and liquid viscosity, is formulated from the Equations. The governing equations of the motion of the liquid and gas phases are the conservation of mass and momentum, which can be expressed as follows

$$
\left\{\begin{array}{c}
\nabla \cdot \breve{U}_{i}^{\mathrm{v}}=0 \\
\left(\frac{\partial}{\partial t}+U_{i} \nabla\right) \stackrel{\mathrm{V}}{U}_{i}=-\frac{\nabla P_{i}}{\rho_{i}}+v_{i} \nabla^{2} U_{i}^{\mathrm{v}}
\end{array}\right.
$$

where the subscript $i=l, g$ denotes the liquid phase and the gas phase, respectively, 
The solutions of Eq.(1) have to satisfy dynamic boundary conditions

$$
\begin{aligned}
& \left\{\begin{array}{l}
p_{r r}=p_{\sigma} \\
p_{r z}=0
\end{array}, r=r_{0}\right. \\
& \left\{\begin{array}{l}
p_{z z}=p_{\sigma}+p_{g} \\
P_{r z}=0
\end{array}, r=r_{0}\right. \\
& \left\{\begin{array}{l}
\mathrm{v}_{g z} \rightarrow u^{g} \\
\mathrm{v}_{g r} \rightarrow 0
\end{array}, r \rightarrow \infty\right. \\
& \left\{\begin{array}{l}
\mathrm{v}_{g r}=\mathrm{v}_{r} \\
\mathrm{v}_{g z}=\mathrm{v}_{z}
\end{array}, r \rightarrow r_{0}\right.
\end{aligned}
$$

The dispersion is derived eventually as follows

$$
\begin{aligned}
& \omega^{2}+\frac{2 v k^{2}}{I_{0}\left(k r_{0}\right)}\left[I_{0}\left(k r_{0}\right)-\frac{I_{1}\left(k r_{0}\right)}{k r_{0}}-\frac{2 k l}{k^{2}+l^{2}} \cdot \frac{I_{1}\left(k r_{0}\right)}{I_{1}\left(l r_{0}\right)}\left(I_{0}\left(l r_{0}\right)-\frac{I_{1}\left(l r_{0}\right)}{l r_{0}}\right)\right] \omega= \\
& \frac{\sigma k}{\rho_{l} r_{0}^{2}}\left(1-k^{2} r_{0}^{2}\right) \cdot \frac{I_{1}\left(k r_{0}\right)}{I_{0}\left(k r_{0}\right)} \frac{l^{2}-k^{2}}{l^{2}+k^{2}}+\frac{\rho_{g} k^{2}\left(u^{g}\right)^{2}}{\rho_{l}} \cdot \frac{K_{0}\left(k r_{0}\right)}{K_{1}\left(k r_{0}\right)} \cdot \frac{I_{1}\left(k r_{0}\right)}{I_{0}\left(k r_{0}\right)} \cdot \frac{l^{2}-k^{2}}{l^{2}+k^{2}}
\end{aligned}
$$

where $I_{n}, K_{n}$ are, respectively, the modified Bessel functions of the first and second kind. $v=\mu / \rho, \sigma$ is the liquid surface tension and the prime indicates differentiation. Eq.(6) relates the wave growth rate $\omega$ to its wave number $k$, and $l$ is defined by

$$
l^{2}=k^{2}+\frac{\omega}{v}
$$

\section{The explicit solutions}

As the parameter $l$ is still a function of $\omega$, the solution is complicated. But it need to simplify the Eq.(6) to get the explicit solution, which is concise to understand how the key parameters influence the breakup process.

When $x \rightarrow \infty$, Bessel functions can be approximated by

$$
I_{n}(x) \sim \frac{e^{x}}{\sqrt{2 \pi x}}, K_{n}(x) \quad \sqrt{\frac{\pi}{2 x}} e^{-x}
$$

Here, $x=k r_{0}$, as the disturbance wavelength is quite small, the wavenumber is large enough, that is to say, $k r_{0} \geq 1$, Then, Eq.(8) becomes

$$
I_{1}\left(k r_{0}\right) \approx I_{0}\left(k r_{0}\right) \approx \frac{e^{k_{0}}}{\sqrt{2 \pi k r_{0}}}, \mathrm{~K}_{1}\left(k r_{0}\right) \approx K_{0}\left(k r_{0}\right) \approx \sqrt{\frac{\pi}{2 k r_{0}}} e^{-k k_{0}}
$$

And substituting the Bessel functions in Eq.(6) by Eq.(9) yields the result

$$
\omega^{2}+2 v k^{2} \omega\left[1-\frac{1}{k r_{0}}-\frac{2 k l}{k^{2}+l^{2}}\left(1-\frac{1}{l r_{0}}\right)\right]=-\frac{\sigma k^{3}}{\rho_{l}} \frac{l^{2}-k^{2}}{l^{2}+k^{2}}+\frac{\rho_{g} k^{2}\left(u^{g}\right)^{2}}{\rho_{l}} \frac{l^{2}-k^{2}}{l^{2}+k^{2}}
$$


Considering the high velocity of the gas, the wavelength is very small, namely:

$$
k r_{0} \quad 1, l^{2}=k^{2}+\frac{\omega}{v}, l r_{0} \quad 1
$$

Eq.(10) reduces to

$$
\omega^{2}+2 v k^{2} \omega\left(1-\frac{2 k l}{k^{2}+l^{2}}\right)=-\frac{\sigma k^{3}}{\rho_{l}} \frac{l^{2}-k^{2}}{l^{2}+k^{2}}+\frac{\rho_{g} k^{2}\left(u^{g}\right)^{2}}{\rho_{l}} \frac{l^{2}-k^{2}}{l^{2}+k^{2}}
$$

Note this paper mainly focuses on the jets with small viscosity, Eq.(7) can be replaced by

$l^{2} \approx \frac{\omega}{v}$

Then, the explicit solution can be obtained as

$$
\omega=-v k^{2}+\sqrt{v^{2} k^{4}+\frac{\rho_{g} k^{2}\left(u^{g}\right)^{2}-\sigma k^{3}}{\rho_{l}}}
$$

In order to confirm the validity of explicit solution Eq.(13), direct comparison with the previous accurate, numerical solution of $\mathrm{Li}^{[5]}$ in the cases of small viscosity is displayed in Fig. 3.

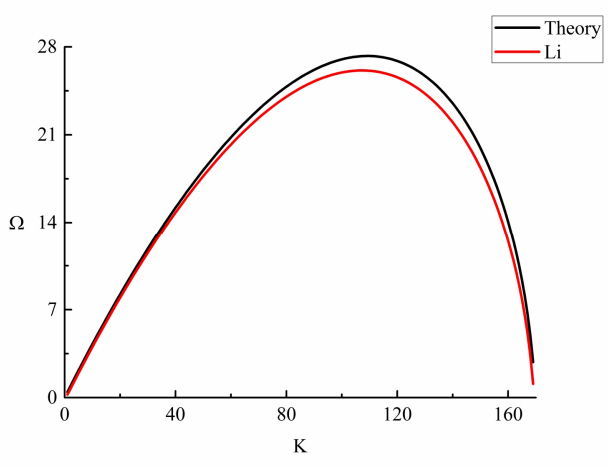

Fig. 3. Numerical solutions curves at viscosity

$$
\mu=0.001 P a / s, r_{0}=1 \mathrm{~mm}, \rho_{l}=1000 \mathrm{~kg} / \mathrm{m}^{3}, \rho_{g}=1.24 \mathrm{~kg} / \mathrm{m}^{3}, u^{g}=100 \mathrm{~m} / \mathrm{s}
$$

It should be noted the two dispersion curves are agreed well in the large and small wavenumber region, while in the regime of the peaks, the explicit solution are larger than the numerical ones to a small extent. To better understand the small distinctions, the relative error, i.e. the ratio of the absolute differences to the accurate numerical solution, in the wavenumber corresponding to the large growth rate is illustrated in Fig. 4. 


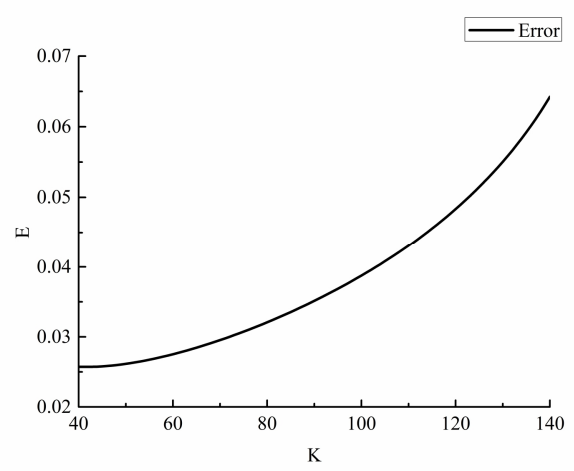

Fig. 4 The relative error diagram

Obviously, the relative error is so small, less than $10 \%$ that can be neglected rationally. As a whole, the explicit solution is a good approximation to the numerical ones and can be used to describe the breakup mechanism of small viscous liquid jets.

\section{Conclusions}

Owing to the complexity of the dispersion equation, the numerical solution has been taken to analyze the principle of the liquid jet breakup. However, the numerical solution fails to tell the role of the different physical parameters in the process. In contrast, the present explicit solution is expressed as functions of gas velocity, liquid velocity and other physical characteristics. This paper proposes an approach to get the explicit solution on the small viscosity liquid jet in high velocity air stream .Agreement between the present explicit solutions and numerical solutions is obtained. Results verify the reliability of the present approach, the accuracy decreases when the liquid viscosity increases.

\section{References}

[1] Rayleigh L.[J]. Proceedings of the London mathematical society (1878), 1(1): 4-13

[2] Levich V G, Technica S. Physicochemical hydrodynamics[M]. Englewood Cliffs, NJ: Prentice-hall (1962)

[3] Reitz R D, Bracco F V. [J]. Physics of Fluids (1958-1988), 25(10): 1730-1742 (1982)

[4] Lin S P, Lian Z W. [J]. AIAA Journal, 28(1): 120-126 (1990).

[5] Li X. [J]. Atomization and sprays, 5(1) (1995). 